Original Article

\title{
Comparisons of trunk and knee mechanics during various speeds of treadmill running between runners with and without patellofemoral pain: a preliminary study
}

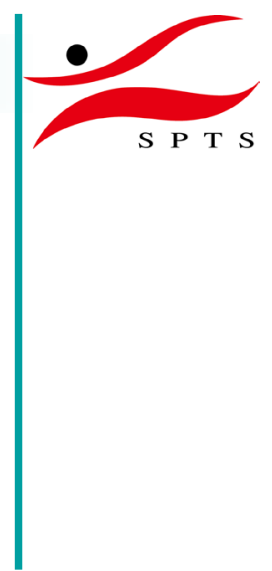

\author{
KaI-Yu Ho, PT, MSPT, PhD ${ }^{1)^{*}}$, Tiffany Barrett, PT, DPT, OCS ${ }^{1)}$, \\ Zachary Clark, PT, DPT ${ }^{1)}$, Christine DuVall, PT, DPT ${ }^{1)}$, Tavin Fox, PT, DPT ${ }^{1}$, \\ Caitlin Howden, PT, DPT ${ }^{1)}$, Andrew Murata, SPT ${ }^{1)}$ \\ 1) Department of Physical Therapy, University of Nevada, Las Vegas: 4505 S. Maryland Parkway, \\ Box 453029, Las Vegas, NV 89154, USA
}

\begin{abstract}
Purpose] To determine if runners with patellofemoral pain (PFP) exhibit higher patellofemoral joint (PFJ) stress and trunk extension compared to pain-free runners during treadmill running. [Participants and Methods] Twelve runners ( 7 with PFP and 5 pain-free) participated in this study. Participants ran at 3 different running conditions: self-selected, fast (120\% of self-selected), and slow ( $80 \%$ of self-selected) speeds. Kinematics and kinetics of trunk and lower extremities were obtained. PFJ stress, PFJ reaction force, and PFJ contact area were determined using a biomechanical model. Two-factor ANOVAs with repeated measures were used to compare outcome variables between 3 speeds and between 2 groups. [Results] There was no significant difference in peak PFJ stress between groups across the 3 speeds. Peak PFJ stress was lowest during slow running compared to fast and selfselected running speed conditions across both groups. No significant difference was found in trunk flexion angle, $\mathrm{PFJ}$ reaction force, or PFJ contact area between groups across the 3 speeds. [Conclusion] Runners with and without PFP exhibited similar peak PFJ stress and trunk flexion angle during treadmill running. This preliminary work does not support the theory that reduced trunk flexion during running contributes to increased PFJ stress in runners with PFP.

Key words: Patellofemoral pain, Trunk flexion angle, Running
\end{abstract}

(This article was submitted May 21, 2021, and was accepted Jul. 21, 2021)

\section{INTRODUCTION}

Patellofemoral joint pain (PFP) is the most prevalent type of injury sustained by runners and accounts for $17 \%$ of musculoskeletal injuries reported in this population ${ }^{1,2)}$. While the cause of PFP is thought to be multifactorial, increased patellofemoral joint (PFJ) stress, defined as PFJ reaction force per unit of contact area, is suggested to be a primary contributing factor for PFP in runners ${ }^{3}$. The recent literature has highlighted the important role of sagittal plane trunk posture on PFJ stress during running in runners with $\mathrm{PFP}^{4}$. Specifically, in healthy runners, running with an upright trunk posture is associated with elevated PFJ stress and reaction forces due to an increased knee extensor moment ${ }^{3,5)}$. The increases in knee extensor moment are mainly driven by an increased knee lever arm (perpendicular distance from the axis of the knee joint to the ground reaction force vector) as a result of a posterior shift of vertical ground reaction force from decreased trunk flexion $^{3)}$. It is further reported that sagittal plane trunk posture can be modified via a treadmill-based, gait re-training program

*Corresponding author. Kai-Yu Ho (E-mail: kaiyu.ho@unlv.edu)

(Supplementary material: refer to PMC https://www.ncbi.nlm.nih.gov/pmc/journals/2193/)

(C2021 The Society of Physical Therapy Science. Published by IPEC Inc.

(c) (1) $\odot$ This is an open-access article distributed under the terms of the Creative Commons Attribution Non-Commercial No Deriva-

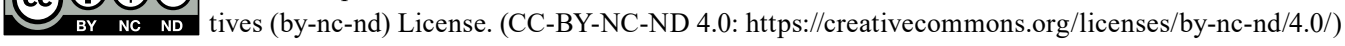


to reduce PFJ stress in asymptomatic runners ${ }^{6}$. However, while an upright trunk posture is suggested to cause high PFJ stresses in pain-free runners, it remains unclear whether runners with PFP would present with a more upright trunk posture and subsequent higher PFJ stresses during running when compared to asymptomatic runners.

In over-ground running, Bazett-Jones et al. ${ }^{7)}$ have demonstrated that runners with and without PFP exhibit similar trunk flexion angle when running at a speed of $4.0 \mathrm{~m} / \mathrm{s}$. However, it should be noted that running mechanics have been found to be different between treadmill and over-ground running ${ }^{8,9)}$. Furthermore, participants often need to accelerate and decelerate while being tested in a limited laboratory space (e.g., 10-m runway) during running, which may affect their trunk posture during running ${ }^{9)}$. Another factor to consider in determining PFJ loading is running speed, as it has been shown that pain-free recreational runners tend to exhibit a higher trunk flexion angle while running over-ground at a faster ${ }^{10,11)}$ or slower speed ${ }^{10)}$.

Taken together, the current evidence of comparing the PFJ mechanics and trunk posture during treadmill running between runners with and without PFP at different speeds is limited. The purpose of this research study was to determine if runners with PFP would exhibit decreased trunk flexion and subsequent elevated PFJ stress during treadmill running when compared to pain-free runners across various speeds. We hypothesized that runners with PFP would present with decreased trunk flexion angle and higher PFJ stress during various speeds (slow, self-selected, and fast) of treadmill running when compared to pain-free runners.

\section{PARTICIPANTS AND METHODS}

We recruited runners between the ages of 18 and 40 years old who ran at least 6 miles/week. The data from an existing study was used to estimate the sample size for detecting changes in sagittal plane trunk posture during running between groups ${ }^{5)}$. With $95 \%$ power, an $\alpha$ level of 0.05 , and a calculated effect size of 2.5 , we estimated that 5 individuals per group would be needed. A total of 12 runners were included for our study: 7 of these individuals had PFP (age $=28.0 \pm 8.2$ years; 5 females and 2 males; weight $=65.2 \pm 12.9 \mathrm{~kg}$; height $=172.7 \pm 12.3 \mathrm{~cm}$; body mass index $[\mathrm{BMI}]=21.7 \pm 2.6)$ and the remaining 5 participants were pain-free (age $=27.4 \pm 7.8$ years; 3 females and 2 males; weight $=75.7 \pm 12.6 \mathrm{~kg}$; height $=175.8 \pm 8.9 \mathrm{~cm}$; $\mathrm{BMI}=24.4 \pm 2.6)$ (Table 1).

The primary inclusion criterion for the PFP group was an insidious onset of retropatellar pain (pain originating from behind the patella) of at least 3 months in duration. Furthermore, participants were screened through physical examination to rule out concomitant sources of pain. This process included palpation of the soft tissues around the PFJ to identify the location of pain. If the source of pain was localized to the quadriceps tendon, patellar tendon, patella bursa, patella fat pad, or tibiofemoral joint, the patient was disqualified from the study. Participants with PFP were excluded from the study if they had prior knee surgery, had a history of traumatic dislocations, were pregnant, or thought they may have been pregnant. To control for potential confounding in running biomechanics, we recruited pain-free runners with similar age, height, weight, and weekly mileage. The pain-free control group had the same inclusion and exclusion criteria as the experimental group, but had no history of PFP within the last 2 years ${ }^{12}$. Prior to participation, all participants were informed of the nature of the study and provided informed consent as approved by the Institutional Review Board of the University of Nevada, Las Vegas (protocol number: 1450804).

A 12-camera motion analysis system (Vicon, Oxford Metrics Ltd., Oxford, UK) was used to capture kinematic data of the lower extremity and trunk at $250 \mathrm{~Hz}$. Ground reaction forces were collected at a sampling rate of 2,000 $\mathrm{Hz}$ using force plates

Table 1. Participant characteristics

\begin{tabular}{lcc}
\hline & PFP group $(\mathrm{n}=7)$ & Control group $(\mathrm{n}=5)$ \\
\hline Age (years) & $28.0 \pm 8.2$ & $27.4 \pm 7.8$ \\
Gender & 5 females; 2 males & 3 females; 2 males \\
Height $(\mathrm{cm})$ & $172.7 \pm 12.3$ & $175.8 \pm 8.9$ \\
Weight $(\mathrm{kg})$ & $65.2 \pm 12.9$ & $75.7 \pm 12.6$ \\
BMI $\left(\mathrm{kg} / \mathrm{m}^{2}\right)$ & $21.7 \pm 2.6$ & $24.4 \pm 2.6$ \\
Weekly mileage (miles/week) & $13.6 \pm 6.7$ & $13.6 \pm 4.7$ \\
AKPS & $81.6 \pm 4.9$ & $\mathrm{~N} / \mathrm{A}$ \\
History of pain (months) & $25.0 \pm 22.7$ & $\mathrm{~N} / \mathrm{A}$ \\
Self-selected running speed $(\mathrm{m} / \mathrm{s})$ & $2.6 \pm 0.6$ & $2.5 \pm 0.5$ \\
Slow running speed $(\mathrm{m} / \mathrm{s})$ & $2.1 \pm 0.4$ & $2.1 \pm 0.4$ \\
Fast running speed $(\mathrm{m} / \mathrm{s})$ & $3.1 \pm 0.7$ & $3.0 \pm 0.7$ \\
\hline
\end{tabular}

No statistically significant difference was observed between the PFP and control groups in age, weight, height, BMI, weekly mileage, and 3 running speeds using independent $\mathrm{t}$-tests $(\mathrm{p}>0.05)$.

BMI: body mass index; AKPS: The Anterior Knee Pain Scale; PFP: patellofemoral pain. 
instrumented in a dual-belt treadmill (Fully Instrumented Treadmill, Bertec Corp., Columbus, OH, USA).

Participants attended one biomechanical testing session. Prior to the testing session, the same investigators placed markers on the trunk and lower extremities. The detailed marker definition has been reported in our previous work ${ }^{3)}$. In brief, anatomical markers were placed on the following anatomical landmarks: the great toe, 1 st and 5th metatarsal heads, medial and lateral malleoli, medial and lateral femoral epicondyles, L5-S1 joint space, greater trochanters, iliac crests, anterior superior iliac spines (ASIS), acromioclavicular joints, and posterior superior iliac spines (PSIS). Additional tracking markers were attached on participant's lateral thigh, lateral leg, and heel counters bilaterally, as well as the spinous process of T3. A standing calibration trial was first obtained to define the segmental coordinate systems and joint axes. After the calibration trial, all anatomical markers were removed except for those at the iliac crests and L5-S1 junction. The tracking markers remained on the participant throughout the running trials.

Participants were then tested under 3 different running conditions: self-selected, fast, and slow speeds. Each participant was first asked to run at his or her self-selected speed on a level treadmill ( 0 degrees of inclination). Once the self-selected speed was determined, fast ( $120 \%$ of self-selected) and slow ( $80 \%$ of self-selected) speeds were estimated. Each participant was tested in the self-selected speed condition first and followed by either slow or fast speed conditions in an alternated order. A 5-minute warm-up session was provided prior to each running condition allowing them to run at their comfortable speed. During each of the running conditions (self-selected, fast, and slow speeds), participants ran for 3 minutes and 3 continuous 20 -second trials were collected during the middle 1-minute. Participants were given a 3-minute rest period between conditions to avoid fatigue.

Vicon Nexus software (Oxford Metric Ltd., Oxford, UK) was utilized to label and digitize the reflective markers used to gather the kinematic data. The ground reaction forces were normalized to participants' body mass for data analysis. The sagittal plane kinematics and kinetics of the trunk and lower extremities were computed using Visual 3D software (C-Motion, Germantown, MD, USA). The trunk segment was defined by markers placed bilaterally on iliac crests and acromioclavicular joints $^{3,5)}$. The trunk and pelvis were modeled as a cylinder, and the lower extremity segments were modeled as a cone ${ }^{3,5)}$. The trunk flexion angle was calculated as the orientation of the trunk segment relative to the global coordinate system of the laboratory ${ }^{3,5)}$.

A previously developed 2-dimensional PFJ model was used to estimate PFJ stress during running. The quadriceps force was estimated by dividing knee extensor moment by the quadriceps moment arm during the running trials. Next, a ratio reported by van Eijden et al. ${ }^{13)}$, showing a relationship between PFJ reaction force and quadriceps force as a function of knee flexion angle, was used to estimate the PFJ reaction force. The last step of the algorithm was to calculate the PFJ stress, which was estimated by dividing PFJ reaction force by the PFJ contact area from the data of Powers et $\mathrm{al}^{14)}$.

The primary variables were peak PFJ stress and trunk flexion angle at the time of peak PFJ stress. The secondary variables were PFJ reaction force and PFJ contact area reaction force at the time of peak PFJ stress. We compared each variable between groups across the 3 conditions using a 2 (groups: PFP and controls) $\times 3$ (speeds: self-selected, fast, and slow) ANOVA with repeated measures. If a significant group-by-speed interaction or a significant main effect was found, post hoc testing (t-tests with a Bonferroni correction) was employed. All statistical analyses were performed with the use of SPSS 24.0 statistical software (International Business Machines Corp, Armonk, NY, USA). A significant difference was defined as p smaller than 0.05 .

\section{RESULTS}

Across the 3 running conditions, the peak PFJ stress occurred at approximately $38 \%$ of the stance phase for the control group and $37 \%$ of the stance phase for the PFP group. There was not a group-by-speed interaction in peak PFJ stress $(p=0.917)$ and no main effect of group in peak PFJ stress during running was found $(p=0.660)$. However, there was a statistically significant main effect of speed on peak PFJ stress $(p=0.006)$. In post hoc analyses, peak PFJ stress was observed to be significantly lower during slow running than at self-selected $(\mathrm{p}=0.002)$ and fast running $(\mathrm{p}=0.017)$ speeds across both groups. There was no significant difference in peak PFJ stress between fast and self-selected running speed conditions $(p=0.435)$ (Table 2).

There was not a group-by-speed interaction in trunk flexion angle at the time of peak PFJ stress $(p=0.540)$. We did not observe a main effect of group $(\mathrm{p}=0.615)$ or main effect of speed $(\mathrm{p}=0.375)$ on trunk flexion angle at the time of peak PFJ stress (Table 2).

There was not a group-by-speed interaction in PFJ reaction force at the time of peak PFJ stress $(\mathrm{p}=0.707)$. There was no main effect of group in PFJ reaction force at the time of peak PFJ stress $(p=0.220)$. We did observe a statistically significant main effect of speed on PFJ reaction force at the time of peak PFJ stress $(\mathrm{p}=0.008)$. In post hoc testing, we observed that PFJ reaction force at the time of peak PFJ stress was significantly lower during slow running than at self-selected $(\mathrm{p}=0.003)$ and fast running $(p=0.020)$ speeds across both groups. There was no significant difference in peak PFJ reaction force at the time of peak PFJ stress between fast and self-selected running speed conditions ( $p=0.507$ ) (Table 2).

There was not a group-by-speed interaction in PFJ contact area at the time of peak PFJ stress ( $\mathrm{p}=0.925)$. We did not observe a main effect of group on PFJ contact area at the time of peak PFJ stress $(p=0.051)$. There was also no main effect of speed on PFJ contact area at the time of peak PFJ stress $(p=0.306)$ (Table 2). 
Table 2. Summary of variables of interest in runners with PFP and pain-free control runners at slow, self-selected, and fast running speeds

\begin{tabular}{|c|c|c|c|c|}
\hline & & $\begin{array}{c}\text { Slow } \\
\text { speed }\end{array}$ & $\begin{array}{l}\text { Self-selected } \\
\text { speed }\end{array}$ & $\begin{array}{c}\text { Fast } \\
\text { speed }\end{array}$ \\
\hline \multirow[t]{3}{*}{ Peak patellofemoral joint stress (MPa) } & PFP & $11.8 \pm 4.9$ & $13.3 \pm 4.8$ & $13.9 \pm 5.3$ \\
\hline & Control & $10.9 \pm 2.6$ & $12.3 \pm 2.7$ & $12.6 \pm 3.2$ \\
\hline & Averaged across 2 groups & $11.4 \pm 3.9$ & $12.9 \pm 4.0^{\dagger}$ & $13.4 \pm 4.4^{\dagger}$ \\
\hline \multirow[t]{2}{*}{ Trunk flexion angle at the time of peak stress $\left(^{\circ}\right)$} & PFP & $3.9 \pm 3.9$ & $4.0 \pm 3.7$ & $4.5 \pm 3.0$ \\
\hline & Control & $5.9 \pm 4.7$ & $4.5 \pm 5.1$ & $5.9 \pm 6.7$ \\
\hline \multirow{3}{*}{$\begin{array}{l}\text { Patellofemoral joint reaction force at the time of } \\
\text { peak stress }(\mathrm{N} / \mathrm{kg})\end{array}$} & PFP & $40.5 \pm 11.1$ & $46.0 \pm 10.0$ & $48.4 \pm 11.1$ \\
\hline & Control & $33.5 \pm 12.7$ & $38.0 \pm 13.8$ & $38.3 \pm 11.0$ \\
\hline & Averaged across 2 groups & $37.6 \pm 11.8$ & $42.6 \pm 11.9^{\dagger}$ & $44.2 \pm 11.8^{\dagger}$ \\
\hline \multirow{2}{*}{$\begin{array}{l}\text { Patellofemoral joint contact area at the time of } \\
\text { peak stress }\left(\mathrm{mm}^{2}\right)\end{array}$} & PFP & $232.6 \pm 2.1$ & $233.1 \pm 2.9$ & $233.8 \pm 3.6$ \\
\hline & Control & $223.9 \pm 10.7$ & $223.8 \pm 10.8$ & $224.9 \pm 10.3$ \\
\hline
\end{tabular}

Data indicated as mean \pm SD.

$\dagger$ indicates a significant difference from the slow running condition $(\mathrm{p}<0.05)$.

PFP: patellofemoral pain.

\section{DISCUSSION}

To our knowledge, this is the first study assessing trunk flexion angle and PFJ loading during various speeds of treadmill running in pain-free runners and runners with PFP. The primary purpose of the study was to compare trunk flexion angle and peak PFJ stress between runners with and without PFP across 3 different speed conditions (self-selected, fast, and slow speeds). Our findings did not support the hypothesis that runners with PFP would have a decreased trunk flexion angle and increased peak PFJ stress when compared to pain-free runners during treadmill running. We found that there was no difference in peak PFJ stress or trunk flexion angle at the time of peak PFJ stress across the 3 running speed conditions between runners with PFP and pain-free runners.

While the mechanism of PFP is complex and multifactorial, decreased trunk flexion, and subsequent increases in knee extensor moment and PFJ reaction force during functional activities are thought to be the contributing factors leading to elevated $\mathrm{PFJ}$ stress and development of $\mathrm{PFP}^{4}$ ). Previous research showed that asymptomatic runners who are cued to demonstrate an upright trunk posture while running show increased PFJ stress and, conversely, exhibit decreased PFJ stress when are cued to demonstrate a more flexed trunk posture ${ }^{5}$. This finding indicates that a more extended trunk posture during running may be one of the factors contributing to elevated PFJ stress and subsequent PFP in runners with PFP. In agreement with the overground running study conducted by Bazett-Jones et al. ${ }^{7)}$, our studies showed that runners with PFP and asymptomatic runners exhibited similar trunk flexion angle during running. Based on the existing literature and our work, these findings question the role of sagittal plane trunk posture in contributing to PFJ stress and PFJ symptoms during running in runners with PFP.

It should be noted that the 2-dimensional PFJ model used in this study only considers sagittal plane parameters of the trunk and knee, which may fail to detect joint stress differences between groups. It has been reported in the existing literature that runners with PFP often present with excessive femoral internal rotation and adduction, ultimately leading to excessive knee valgus during running $\left.{ }^{15}, 16\right)$. Using subject-specific finite element models that consider 3-dimensional kinematics of the hip and knee, and 3-dimensional geometry of the femur and patella, Liao and Powers ${ }^{17}$ ) have found that excessive femoral adduction and internal rotation in runners with PFP result in increased PFJ cartilage stress when compared to pain-free runners. As such, the PFJ symptoms and elevated PFJ stress during running in runners with PFP may be mainly driven by the faulty movement patterns of the hip and knee in the frontal and transverse planes, rather than the trunk posture in the sagittal plane.

Our study did not show a difference in sagittal plane trunk posture between 3 running speeds across both groups, which was not consistent with the findings reported by Fisher et al. ${ }^{10)}$ who showed that asymptomatic runners increase their trunk flexion in both slow and fast running conditions during over-ground running. Such differences may be explained by different running environments used between our study (treadmill) and the previous work (over-ground). In fact, it has been shown that the sagittal plane trunk posture remains unchanged in treadmill running during acceleration while the trunk leans forward during acceleration of over-ground running. This occurs because, in treadmill running, there is no need to account for the linear whole-body inertia that is required for over-ground running when changing speeds ${ }^{9}$. In addition to the findings by Fisher et al. ${ }^{10)}$, Preece and colleagues' study ${ }^{11)}$ showed that recreational runners increase trunk flexion with increased running speed during over-ground running. One notable difference between Preece et al's work ${ }^{11}$ and our study, besides use of overground running, is that their participants ran at various speeds between $3.3 \mathrm{~m} / \mathrm{s}$ and $5.6 \mathrm{~m} / \mathrm{s}^{11)}$ whereas the average running speeds in our study were only between $2.1 \mathrm{~m} / \mathrm{s}$ and $3.0 \mathrm{~m} / \mathrm{s}$ for controls and between $2.1 \mathrm{~m} / \mathrm{s}$ and $3.1 \mathrm{~m} / \mathrm{s}$ for participants with PFP. It is postulated that lower speed ranges (between about 2 and $4 \mathrm{~m} / \mathrm{s}$ ) may not be sufficient to significantly affect 
thoracic kinematics during running ${ }^{10)}$. Taken together, different running terrains (treadmill versus over-ground) and running speed ranges (low versus high) utilized between our work and the existing literature may have contributed to such different findings in trunk kinematics during running.

With respect to the findings of this study, one primary limitation needs to be recognized. Although our study contained the necessary participant number to meet the pre-calculated sample size, a larger sample size may yield a better understanding of the running mechanics in this population. Nevertheless, the important experimental findings observed in our preliminary work may provide clinical insights into the management of runners with PFP.

In conclusion, our study suggests that runners with and without PFP exhibited similar peak PFJ stress and trunk flexion angle when running at slow, self-selected, and fast speeds on a treadmill. The clinical relevance of this preliminary work does not support the theory that reduced trunk flexion during running is a contributing factor to increased PFJ stress in runners with PFP.

\section{Funding}

This work was supported by University of Nevada, Las Vegas Physical Therapy Student Opportunity Research Grant (SORG).

\section{Conflict of interest}

The authors have no conflicts of interest relevant to this article.

\section{REFERENCES}

1) Francis $\mathrm{P}$, Whatman $\mathrm{C}$, Sheerin $\mathrm{K}$, et al.: The proportion of lower limb running injuries by gender, anatomical location and specific pathology: a systematic review. J Sports Sci Med, 2019, 18: 21-31. [Medline]

2) Walter SD, Hart LE, McIntosh JM, et al.: The Ontario cohort study of running-related injuries. Arch Intern Med, 1989, 149: 2561-2564. [Medline] [CrossRef]

3) Ho KY, French T, Klein B, et al.: Patellofemoral joint stress during incline and decline running. Phys Ther Sport, 2018, 34: 136-140. [Medline] [CrossRef]

4) Powers CM, Witvrouw E, Davis IS, et al.: Evidence-based framework for a pathomechanical model of patellofemoral pain: 2017 patellofemoral pain consensus statement from the 4th International Patellofemoral Pain Research Retreat, Manchester, UK: part 3. Br J Sports Med, 2017, 51: 1713-1723. [Medline] [CrossRef]

5) Teng HL, Powers CM: Sagittal plane trunk posture influences patellofemoral joint stress during running. J Orthop Sports Phys Ther, 2014, 44: 785-792. [Medline] [CrossRef]

6) Teng HL, Dilauro A, Weeks C, et al.: Short-term effects of a trunk modification program on patellofemoral joint stress in asymptomatic runners. Phys Ther Sport, 2020, 44: 107-113. [Medline] [CrossRef]

7) Bazett-Jones DM, Cobb SC, Huddleston WE, et al.: Effect of patellofemoral pain on strength and mechanics after an exhaustive run. Med Sci Sports Exerc, 2013, 45: 1331-1339. [Medline] [CrossRef]

8) Bailey J, Mata T, Mercer JA: Is the relationship between stride length, frequency, and velocity influenced by running on a treadmill or overground? Int J Exerc Sci, 2017, 10: 1067-1075. [Medline]

9) Caekenberghe IV, Segers V, Aerts P, et al.: Joint kinematics and kinetics of overground accelerated running versus running on an accelerated treadmill. J R Soc Interface, 2013, 10: 20130222. [Medline] [CrossRef]

10) Fisher D, Louw Q, Cockcroft J, et al.: Three-dimensional kinematics of the thorax during over-ground running. J Bodyw Mov Ther, 2018, 22: 300-307. [Medline] [CrossRef]

11) Preece SJ, Mason D, Bramah C: How do elite endurance runners alter movements of the spine and pelvis as running speed increases? Gait Posture, 2016, 46: 132-134. [Medline] [CrossRef]

12) Ho KY, Hu HH, Colletti PM, et al.: Recreational runners with patellofemoral pain exhibit elevated patella water content. Magn Reson Imaging, 2014, 32: 965-968. [Medline] [CrossRef]

13) van Eijden TM, Weijs WA, Kouwenhoven E, et al.: Forces acting on the patella during maximal voluntary contraction of the quadriceps femoris muscle at different knee flexion/extension angles. Acta Anat (Basel), 1987, 129: 310-314. [Medline] [CrossRef]

14) Powers CM, Lilley JC, Lee TQ: The effects of axial and multi-plane loading of the extensor mechanism on the patellofemoral joint. Clin Biomech (Bristol, Avon), 1998, 13: 616-624. [Medline] [CrossRef]

15) Willy RW, Davis IS: The effect of a hip-strengthening program on mechanics during running and during a single-leg squat. J Orthop Sports Phys Ther, 2011, 41: 625-632. [Medline] [CrossRef]

16) Davis IS, Tenforde AS, Neal BS, et al.: Gait retraining as an intervention for patellofemoral pain. Curr Rev Musculoskelet Med, 2020, 13: 103-114. [Medline] [CrossRef]

17) Liao TC, Powers CM: Tibiofemoral kinematics in the transverse and frontal planes influence the location and magnitude of peak patella cartilage stress: an investigation of runners with and without patellofemoral pain. Clin Biomech (Bristol, Avon), 2019, 62: 72-78. [Medline] [CrossRef] 